\title{
Calcitonin and bone formation: a knockout full of surprises
}

\author{
Mone Zaidi, Baljit S. Moonga, and Etsuko Abe \\ Mount Sinai Bone Program, Mount Sinai School of Medicine, and The Bronx Veterans Affairs Geriatrics Research, \\ Education and Clinical Center, New York, New York, USA
}

J. Clin. Invest. 110:1769-1771 (2002). doi:10.1172/JCI200217425.

\section{Historical perspectives}

In this issue of the JCI, the elegant publication by Hoff and colleagues (1), by serendipity or on purpose, falls on the 40th anniversary of the discovery of calcitonin (CT) as a hypocalcemic principle from the parathyroid gland (2). The parathyroid origin of calcitonin was revisited shortly thereafter, and a thyroid $\mathrm{C}$ cell origin was firmly established ( 3 , 4). Calcitonins from several species mammals, birds, and fishes - were subsequently purified (5), and the first of many calcitonin receptors was cloned in 1991 (6). Tissue-specific alternative splicing of the calcitonin gene at exon 4 was shown to result in production of a novel 37-amino acid peptide: calcitonin gene-related peptide- $\alpha$ (CGRP- $\alpha)$ (7). Both CGRP and calcitonin date back to their neural origins in protochordates and primitive chordates (8). Nonetheless, while CGRP is an established vasodilator and neuromodulator $(8,9)$, calcitonin has had a checkered history, to say the least, both as a hormone and as a drug. A novel effect on bone formation (1), direct or indirect, is thus intriguing and, if proven, will surely add another historical dimension to calcitonin's role in skeletal biology.

\footnotetext{
Address correspondence to: Mone Zaidi, P.O. Box 1055, Endocrinology, Mount Sinai School of Medicine, One Gustave L. Levy Place, New York, New York 10029, USA. Phone: (212) 241-8798; Fax: (212) 426-8312; E-mail: mone.zaidi@msnyuhealth.org. Conflict of interest: Mone Zaidi is a speaker for Proctor and Gamble, Merck, Eli Lilly, and Novartis. Work in the laboratory is also supported by Proctor and Gamble.

Nonstandard abbreviations used: calcitonin (CT); calcitonin gene-related peptide (CGRP); parathyroid hormone $(\mathrm{PTH})$
}

\section{Molecular mechanism of calcitonin action}

Calcitonin is a 32-amino acid peptide with an $\mathrm{N}$-terminal disulphide bridge and a C-terminal prolineamide residue. Both ends of the molecule contain species-invariant residues that are required for its binding to highaffinity $G$ protein-coupled receptors on the osteoclast (5). Attempts to map the entire bimolecular surface of the hormone receptor complex through photolabeling studies have yielded important new information on contact sites (10). It has also become clear that conformational flexibility of a given calcitonin molecule is the primary determinant of its biological potency (5). Thus, the more flexible fish calcitonins (eel and salmon), containing amino acids such as Gly with less bulky side chains, are about 40fold more potent than mammalian (porcine, ovine, and human) homologs $(11,12)$. The ligand specificity of a particular calcitonin receptor isoform is defined by receptor activity-modifying proteins (RAMPs) that form heterodimers with a pair of receptors (13). Femtomolar calcitonin concentrations inhibit the resorptive function of mature osteoclasts, with quiescence ( $\mathrm{Q}$ effect) being followed by margin retraction (R effect) (14). These kinetically separable morphological changes, $\mathrm{Q}$ and $\mathrm{R}$, are exerted through separate CAMP- and $\mathrm{Ca}^{2+}$-dependent signaling pathways involving distinct G proteins (14-17).

\section{Physiological significance}

The physiological significance of calcitonin in skeletal conservation has been challenged, despite clear evidence of osteoclastic sensitivity to the peptide in vitro (12). This is because it has been difficult to explain why thyroidectomy does not result in osteoporosis, and why high circulating calcitonin in medullary thyroid carcinoma fails to trigger overt osteopetrosis. At the cellular level, the mechanism is simple. Calcitonin inhibits extracellular $\mathrm{Ca}^{2+}$ sensing (18), a potent antiresorptive signal, and by implication, calcitonin withdrawal should enhance $\mathrm{Ca}^{2+}$ sensing and limit resorption. Basal resorption is likewise unaffected in the CT/CGRP-null mouse (1). A key finding, that calcitonin withdrawal sensitizes an osteoclast to parathyroid hormone-induced (PTH-induced) stimulation, witnessed by elevations in serum calcium and urinary deoxypyridinoline cross-links, most certainly establishes its physiological function in resorption control. In humans, serum calcitonin rises during pregnancy, growth, and lactation (19). It is during these periods of calcium stress that a tonic antiresorptive hormone will best exert its effect to limit skeletal loss. That, we are now led to believe (1), may be the primary role of calcitonin in skeletal conservation.

What is impressive, albeit unexpected and difficult to explain, is the osteosclerotic phenotype in the CT/CGRP-null mouse described by Hoff et al. (1) (Figure 1). The dramatic increase in the rate of bone formation, evident from tetracycline labeling studies (1), appears to counteract (and abolish) the bone loss due to ovariectomy. A direct role for calcitonin in this effect, however, seems unlikely. According to the authors, there is little evidence of an osteoblastic calcitonin receptor, and it is unlikely that an osteoclast-derived molecule signals the increased bone formation. Growth factors released from the 


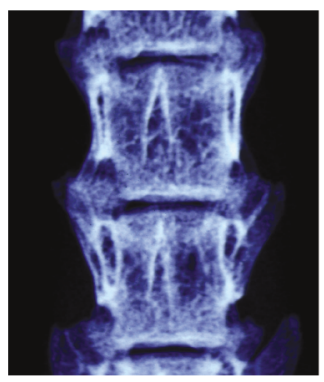

$+/+$

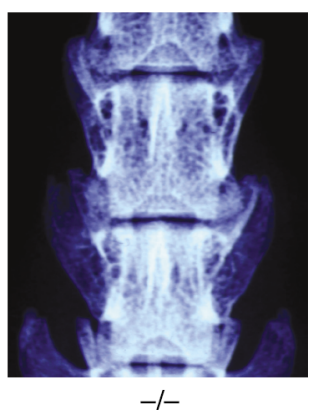

Figure 1

Radiographic analysis of the vertebrae of 3-month-old female wild-type and CT/CGRPdeficient mice (1).

bone matrix could be potential mediators, but again, basal resorption in these animals remains normal. This also rules out the possibility that the increase in bone formation is due to a tight coupling between formation and resorption, as occurs following ovariectomy.

Indeed, the increased bone formation appears to be predominant and independent of resorption (1). Speculatively, we suggest that the effects of CT/CGRP withdrawal are exerted centrally via leptin. The enhanced bone formation in the leptin receptor-deficient $d b / d b$ mouse is identical to that seen in the CT/CGRP-null mouse (20). Even more interesting is the recent revelation that the sympathetic nervous system, regulated by leptin, may control bone formation through adrenergic receptors on osteoblasts (21). Furthermore, several neuropeptides, such as neuropeptide $\mathrm{Y}$, have recently been shown to exert unexpected but powerful effects on the skeleton (22). CGRP is localized to cells in close proximity to leptinergic neurons (23), and it would not be surprising, therefore, if the leptin axis were modulated by CGRP directly or indirectly. CGRP- $\beta$, the product of a second CGRP gene (5), should, however, at least in part, compensate for the loss of CGRP- $\alpha$, and this requires further investigation using double knockout approaches.

\section{Calcitonin as a drug?}

While the CT/CGRP-null phenotype points out that there is still much about the physiological effects of calcitonin and its role in bone remodeling that we do not understand, decades of application in humans have taught us a great deal about its therapeutic utility. Calcitonin has been used for Paget bone disease, hypercalcemia of malignancy, osteogenesis imperfecta, and postmenopausal osteoporosis. Both subcutaneous and nasal calcitonin formulations have been developed and are in use worldwide. There is rich anecdotal evidence that subcutaneous calcitonins, namely salmon, eel, porcine, and human calcitonins, all significantly increase bone mineral density and, in doing so, likely reduce fracture risk (24). However, to the contrary, there is evidence that the effects of the currently available nasal salmon-calcitonin formulation in reducing fracture risk are minimal, mainly due to issues of bioavailability (25). This is not unexpected, because like other highly charged peptides, including insulin and PTH, calcitonin is unlikely to cross multicellular mucous membranes effectively in the absence of a vehicle to aid its delivery.

The widespread use of calcitonin as a nasal spray is thus likely to be re-evaluated (26). Evolving therapies, including newer bisphosphonates, have impinged and would continue to impinge upon calcitonin's success as an antiosteoporosis therapeutic (Table 1). Nonetheless, two key advantages of calcitonin would remain should the desired bioavailability be achieved. One is its stabilizing effect on bone microarchitecture, which precedes changes in bone mineral density (25). This may account for its rapid effect in reducing fracture risk, also seen with the newer bisphosphonates (27). Second and perhaps more important is the use of the peptide in achieving analgesia in vertebral crush syndromes and other types of osteogenic pain.

Finally, with the revelation that lowdose intermittent PTH reduces fracture risk through a dramatic increase in bone formation (28), the possibility of sequential therapy with the two hormones, PTH and calcitonin, makes solid therapeutic sense. The paper by Hoff and colleagues (1), however, raises an important question: Can systemically administered calcitonin inhibit bone formation and thus counteract the anabolic effect of PTH in humans? Although evidence suggests that this is not the case, future systematic clinical studies should clarify this one way or another.

\section{Table 1}

Osteoporosis therapeutics on the horizon

\section{Antiresorptive}

Bisphosphonates ${ }^{A}$, including new potent intravenous analogues Selective sex steroid (estrogen ${ }^{A}$ and androgen) receptor modulators Calcitonin ${ }^{\mathrm{A}}$, calcitonin analogues, and calcitonin secretagogues

Future therapies targeted at inhibiting osteoclast formation and function

Osteoprotegerin and its analogues

RANK and RANK-ligand inhibitors

Specific cytokine inhibitors and receptor modulators

Cathepsin K inhibitors

Integrin inhibitors

Vacuolar $\mathrm{H}^{+}$ATPase (proton pump) inhibitors

\section{Bone forming}

Parathyroid hormone and its analogues

Parathyroid hormone secretagogues (calcium receptor antagonists)

Anabolic vitamin D analogues

Modifiers of the bone morphogenetic protein cascade, including new statins

Centrally acting bone formation modulators

${ }^{A}$ Established therapies. 


\section{The future}

The report by Hoff et al. (1) not only establishes a role for calcitonin in skeletal conservation, expected since its discovery four decades ago, but also opens new areas of investigation. These investigations extend from examining a role for calcitonin and CGRP in the central control of bone remodeling to defining other, perhaps more novel functions of both peptides, considering that their receptors have a widespread distribution (5).

On the therapeutic front, calcitonin has stood the test of time. Nonetheless, several new approaches are being used to enhance calcitonin access to the osteoclast, and, for its longer-term use in humans, to prevent receptor downregulation. The next decade should thus witness the development of oral formulations, new peptide delivery systems, and membrane-permeant analogs with in vivo potencies suggested by calcitonin's powerful cellular effects $(29,30)$. A full characterization of the calcitonin receptor has allowed the synthesis of isoform-specific and even domain-specific calcitonin analogs. Likewise, organic molecules that could allosterically enhance receptor sensitivity to circulating calcitonin constitute possible future therapeutics. Finally, one could envisage the development of a calcitonin secretagogue a small-molecule agonist targeted to the $\mathrm{C}$ cell $\mathrm{Ca}^{2+}$ receptor that would activate calcitonin release, as has been achieved successfully for PTH $(31,32)$.

\section{Acknowledgments}

M. Zaidi acknowledges support of the $\mathrm{NIH}$ (National Institute on Aging) and the US Department of Veterans Affairs.
1. Hoff, A.O., et al. 2002. Increased bone mass is an unexpected phenotype associated with deletion of the calcitonin gene. J. Clin. Invest. 110:1849-1857. doi:10.1172/JCI200214218.

2. Copp, D., and Cheney, B.A. 1962. Calcitonin: a hormone from the parathyroid that lowers calcium level of the blood. Nature. 193:381-382.

3. Foster, G.V., et al. 1964. Thyroid origin of calcitonin. Nature. 202:1303-1305.

4. Aliapoulios, M.A., Voelkel, E.F., and Munson, P.L. 1966. Assay of human thyroid glands for thyrocalcitonin activity. J. Clin. Endocrinol. Metab. 26:897-901.

5. Zaidi, M., et al. 2002. Forty years of calcitonin: where are we now? A tribute to the work of Iain MacIntyre, FRS. Bone. 30:655-663.

6. Lin, H.Y., et al. 1991. Expression cloning of an adenylate cyclase-coupled calcitonin receptor. Science. 254:1022-1024.

7. Rosenfeld, M.G., et al. 1983. Production of a novel neuropeptide encoded by the calcitonin gene via tissue-specific RNA processing. Nature. 304:129-135.

8. Girgis, S.I., et al. 1980. Immunoreactive human calcitonin-like molecule in the nervous systems of protochordates and a cyclostome, Myxine. J. Endocrinol. 87:375-382.

9. Girgis, S.I., et al. 1985. Calcitonin gene-related peptide: potent vasodilator and major product of calcitonin gene. Lancet. 2:14-16.

10. Suva, L.J., et al. 1997. Design, synthesis and utility of novel benzophenone-containing analogs for photoaffinity labeling of the calcitonin receptor. J. Pharmacol. Exp. Ther. 283:876-888

11. Kapurniato, A., and Taylor, J.W. 1995. Structura and conformational requirements for human calcitonin activity. Design, synthesis and study of lactam bridge analogs. J. Med. Chem. 38:836-847.

12. Zaidi, M., et al. 1987. Calcitonin gene-related peptide inhibits osteoclastic bone resorption: a comparative study. Calcif. Tissue Int. 4:149-154.

13. Armour, S.L., Foord, S., Kenakin, T., and Chen, W 1999. Pharmacological characterization of receptor-activity-modifying-proteins (RAMPs) and the human calcitonin receptor. J. Pharmacol. Toxicol. Methods. 42:217-224.

14. Zaidi, M., et al. 1990. Evidence that the action of calcitonin on rat osteoclasts is mediated by two $\mathrm{G}$ proteins acting via separate post-receptor pathways. J. Endocrinol. 136:473-481.

15. Moonga, B.S., et al. 1992. Regulation of cytosolic free calcium in rat osteoclasts by calcitonin. J. Endocrinol. 132:241-249.

16. Chakraborty, M., et al. 1991. Cell cycle-dependent coupling of the calcitonin receptor to different $\mathrm{G}$ proteins. Science. 251:1078-1082.

17. Chen, Y., et al. 1998. The calcitonin receptor stimulates shc tyrosine receptor phosphoryla- tion and Erk1/2 activation. Involvement of Gi protein kinase C and calcium. J. Biol. Chem. 273:19809-19816.

18. Zaidi, M., et al. 1996. Regulation of extracellular calcium sensing in rat osteoclasts by femtomolar calcitonin concentrations. Am. J. Physiol. 271:F637-F644.

19. Whitehead, M., et al. 1981. Interrelations of cal cium-regulating hormones during normal pregnancy. Br. Med. J. 283:10-12.

20. Ducy, P., et al. 2000. Leptin inhibits bone formation through a hypothalamic relay: a central control of bone mass. Cell. 100:197-207.

21. Takeda, S., et al. 2002. Leptin regulates bone formation via the sympathetic nervous system. Cell. 111:305-317.

22. Baldock, P.A., et al. 2002. Hypothalamic Y2 receptors regulate bone formation. J. Clin. Invest 109:915-921. doi:10.1172/JCI200214588.

23. Burdyga, G., et al. 2002. Expression of the leptin receptor in rat and human nodose ganglion neurones. Neuroscience. 109:339-347.

24. Reginster, J.Y. 1993. Calcitonin for prevention and treatment of osteoporosis. Am. J. Med. 95:44S-47S.

25. Chestnut, C.H., III, et al. 2000. A randomized tria of nasal spray salmon calcitonin in postmenopausal women with established osteoporosis. The Prevent Recurrence of Osteoporotic Fracture Study. PROOF Study Group. Am. J. Med. 109:267-276.

26. Colman, E., Hedin, R., Swann, J., and Orloff, D 2002. A brief history of calcitonin. Lancet. 359:885-886.

27. Borah, B., et al. 2002. Risedronate preserves trabecular architecture and increases bone strength in vertebra of ovariectomized minipigs as measured by three-dimensional microcomputed tomography. J. Bone Miner. Res. 17:1139-1147.

28. Neer, R.M., et al. 2001. Effect of parathyroid hormone (1-34) on fractures and bone mineral density in postmenopausal women with osteoporosis. N. Engl. J. Med. 344:1434-1441.

29. Dogru, S.T., Calis, S., and Oner, F. 2000. Oral multiple $\mathrm{w} / \mathrm{o} / \mathrm{w}$ emulsion formulation of a peptide, salmon calcitonin: in vitro and in vivo evaluation. J. Clin. Pharm. Ther. 25:435-443.

30. Chang, S.L., Hoffman, G., Deftos, L.J., and Banga, A.K. 2000. Transdermal iontophoretic delivery of salmon calcitonin. Int. J. Pharm. 25:10-13.

31. Goodman, W.G., et al. 2000. A calcimimetic agent lowers plasma parathyroid hormone in patients with secondary hyperparathyroidism. Kidney Int. 58:436-445.

32. Silverberg, S.J., et al. 1997. Short-term inhibition of parathyroid hormone secretion by a calcium receptor agonist in patients with primary hyperparathyroidism. N. Engl. J. Med. 337:1506-1510. 